\title{
Role of re-growth interface preparation process for spectral line-width reduction of single InAs site-controlled quantum dots
}

\author{
Jesús Herranz, Lukasz Wewior, Benito Alén, David Fuster, Luisa González and Yolanda \\ González
}

IMM-Instituto de Microelectrónica de Madrid (CNM-CSIC), Isaac Newton 8, PTM, E-28760 Tres Cantos, Madrid, Spain

jesus@imm.cnm.csic.es

\begin{abstract}
We present growth and optical characterization measurements of single InAs site controlled quantum dots (SCQDs) grown by molecular beam epitaxy on GaAs (001) patterned substrates by atomic force microscopy oxidation lithography. InAs SCQDs directly grown on the patterned surface were used as a seed layer and strain template for the nucleation of optically active single InAs SCQDs. The preservation of initial geometry of the engraved pattern motifs after the regrowth interface preparation process, the lack of buffer layer growth prior to InAs seed layer deposition and the development of suitable growth conditions provide us an improvement of SCQDs active layer optical properties retaining at the same time a high ratio of single occupation (89\%). In this work a fivefold reduction on the average optical line-width from $870 \mu \mathrm{eV}$ to 156 $\mu \mathrm{eV}$ for InAs SCQDs located $15 \mathrm{~nm}$ from the re-growth interface is obtained by increasing the temperature of the initial thermal treatment step of the re-growth interface from $490^{\circ} \mathrm{C}$ to $530^{\circ} \mathrm{C}$.
\end{abstract}

\section{Introduction}

Semiconductor quantum dots (QDs) have attracted great attention due to their potential as building blocks for quantum information technologies. Single photon and entangled photon pair emission from individual semiconductor QD have been demonstrated, using both optical and electrical injection [1-4]. The development of single QD based devices for quantum information technologies imposes strong requirements in the fabrication of QDs. In terms of the spatial location, the development of fabrication schemes of QDs with precise control of the nanostructure location is desirable to enable the deterministic and scalable integration of single 
QDs in practical devices [5]. Different technological approaches for positioning semiconductor quantum dots have been developed, but, among them, those based on epitaxial growth of the nanostructures on nanoholes patterned substrates are the most widely employed [6,7]. Nanoholes, patterned in the substrate surface, act as preferential nucleation sites for selective QD formation. The selectivity arises from the local minima of the surface chemical potential located at the bottom of each nanohole, related to the local curvature, which drives adatom migration towards the nanoholes [8]. Several lithographic techniques have been used for the fabrication of patterned substrates with application in growth of site-controlled quantum dots (SCQDs), including e-beam lithography [9-13], nanoimprint lithography (NIL) [14,15] and atomic force microscopy (AFM) oxidation lithography [16,18]. AFM oxidation lithography enables the direct patterning of the substrates (without the use of resists), provides high positioning accuracy and it is a very promising lithographic technique for the deterministic integration of nanostructures in photonic microcavities and devices [19,20].

Some common epitaxial procedures might have an undesired impact on the patterned features and should be used with caution during epitaxial growth. Conventional thermal oxide desorption at high temperature is not compatible with the growth on patterned substrates due to surface pitting [21]. Instead, other techniques, such as atomic $\mathrm{H}$ treatment [22,23], Ga assisted oxide desorption [24] or special chemical native oxide removal processes [25], are suitable for patterned substrates. Furthermore, growth of thick buffer layers or growth at high temperature should be avoided due to the smoothening of the patterned motifs, in order to preserve the selectivity and control of the QD formation in the nanoholes. These limitations force the positioned nanostructures to be grown close to the air-exposed surfaces where the pattern was fabricated. Degradation of the optical properties has been commonly observed, being usually attributed to defects or contamination in the re-growth interface, introduced during the processes related to patterning of the substrate. Spectral diffusion effects, caused by random carrier trapping in the QD surroundings, produce homogenous broadening of optical emission lines of SCQDs in proximity to interfaces $[26,27]$.

As high optical quality of nanostructures is a crucial requirement for quantum information applications, great effort has been devoted in recent years to improve the optical properties of positioned nanostructures. Optimization of the patterning and cleaning and preparation procedures for epitaxial growth are key steps to reduce the degradation of the optical properties of the SCQD caused by the proximity to the re-growth interface. Increasing the distance between the active nanostructures and the re-growth interface by means of thicker buffer layers[28] or vertical stacking [29] are convenient growth strategies to improve the optical quality of SCQDs. 
Vertical stacking of positioned nanostructures allows to replicate the pattern design and to increase the distance between active SCQD and the re-growth interface. InAs SCQDs nucleation in the active layer is strain driven by the buried InAs QDs from the seed layer. This strategy has been applied successfully to fabricate SCQDs with improved optical properties [30]. Recently, it has been reported the growth of SCQDs whose optical properties could match those of self-assembled QDs [31,32,28]. Despite this encouraging result, reducing the inhomogeneity of the optical and structural properties of the positioned nanostructures remains a challenging task.

In this work we report on the optical properties of single InAs SCQDs grown by molecular beam epitaxy (MBE) on $\operatorname{GaAs}(001)$ patterned substrates, fabricated by AFM oxidation lithography. Thermal annealing of the re-growth interface up to $530^{\circ} \mathrm{C}$ and vertical stacking of the nanostructures provides an improvement of the InAs SCQDs optical properties with respect to our previously studied single layer InAs SCQDs grown on same kind AFM oxidation lithography patterned substrates [33]. Micro-photoluminescence ( $\mu$ PL) measurements of the vertically stacked active InAs SCQDs, located $15 \mathrm{~nm}$ from the re-growth interface reveal an average exciton line-width of $156 \mu \mathrm{eV}$ with a standard deviation of $50 \mu \mathrm{eV}$.

\section{Experimental details}

Nanoholes templates are fabricated by means of AFM oxidation lithography and subsequent chemical removal of fabricated oxide motifs. We use epitaxial GaAs substrates, consisting of $500 \mathrm{~nm}$ thick GaAs layers grown on epi-ready commercial (001) substrates by MBE at a growth rate of $\mathrm{Ga}, \mathrm{r}_{\mathrm{g}}(\mathrm{Ga})=1$ monolayer per second $(\mathrm{ML} / \mathrm{s})$ at substrate temperature $\mathrm{T}_{\mathrm{S}}=580^{\circ} \mathrm{C}$. These substrates are directly patterned by AFM local oxidation, operating a Nanotec commercial AFM system in dynamic mode in ambient conditions $(\mathrm{RH}=20-30 \%)$. Fabricated patterns consist of square arrays of oxide motifs, with dimensions of the oxide base radius in the range $r_{o x}=50$ $120 \mathrm{~nm}$ and $2 \mu \mathrm{m}$ pattern pitch. Nanoholes are formed by selective removal of the fabricated oxides points by a HF wet etching step (HF 50\%, 5 minutes) and immediately introduced in the MBE system. This simple procedure prior to epitaxial re-growth is a noticeable advantage of AFM oxidation lithography, where no resists are used for patterning.

Samples are first degassed at $\mathrm{T}_{\mathrm{S}}=200^{\circ} \mathrm{C}$ for 12 minutes before being transferred to the MBE growth chamber. A 30 minute atomic $\mathrm{H}$ treatment is applied to the samples to remove surface oxides [22] and to obtain a clean surface suitable for epitaxial re-growth [23]. Surface sample is exposed at a substrate temperature $\mathrm{T}_{\mathrm{S}}=450^{\circ} \mathrm{C}$ to atomic $\mathrm{H}$ flux, obtained by thermal cracking of $\mathrm{H}_{2}$, at a base pressure of $\mathrm{H}_{2} \mathrm{P}=4.5 \cdot 10^{-5}$ Torr, with simultaneous As $\mathrm{s}_{4}$ supply at beam equivalent pressure, $\mathrm{BEP}\left(\mathrm{As}_{4}\right)=6.4 \cdot 10^{-6}$ Torr. Once the surface oxide has been removed from the GaAs surface, two alternative growth protocols are followed for single layer InAs SCQD or vertically 
stacked InAs SCQD. For single layer InAs SCQDs, a 30nm buffer layer is grown at low temperature $T_{S}=450^{\circ} \mathrm{C}$ by atomic layer MBE [34] at growth rate $r_{g}(\mathrm{Ga})=0.5 \mathrm{ML} / \mathrm{s}$, with a pulse sequence $1.2 \mathrm{~s}\left(\mathrm{As}_{4} \mathrm{OFF}, \mathrm{Ga} \mathrm{ON}\right) / 0.8 \mathrm{~s}\left(\mathrm{As}_{4} \mathrm{ON}, \mathrm{Ga} \mathrm{ON}\right)$. For vertically stacked InAs SCQDs no buffer layer is grown and InAs will be deposited directly on the re-growth interface.

The growth procedure for InAs deposition is common for single layer SCQDs and vertically stacked InAs SCQDs. This procedure consists of increasing substrate temperature to $T_{S}=530^{\circ} \mathrm{C}$ in an 8 minutes ramp with the As cell closed and then it is lowered to InAs deposition temperature, $\mathrm{T}_{\mathrm{S}}=510^{\circ} \mathrm{C}$. InAs is then deposited at a growth rate $\mathrm{r}_{\mathrm{g}}(\mathrm{In}) \sim 0.01 \mathrm{ML} / \mathrm{s}$ at $\mathrm{As}_{4}$ overpressure, expressed in terms of their equivalence to ML/s of As on a GaAs (001) surface, $\mathrm{F}_{\mathrm{eq}}(\mathrm{As})=0.5 \mathrm{ML} / \mathrm{s}$ [35]. Indium coverage is set to $\sim 1.5 \mathrm{ML}$, below the critical thickness for QD formation on flat surface $(\sim 1.7 \mathrm{ML}$, as observed from $2 \mathrm{D}-3 \mathrm{D}$ transition in the reflecton high energy electron diffraction diagram) to avoid the formation of self-assembled QDs in unpatterned regions. A 2 minutes growth interruption is applied to promote the indium atoms surface migration leading to InAs nucleation in the pattern sites. For the single layer SCQDs, the obtained InAs nanostructures constitutes the active nanostructures, while for vertically stacked SCQDs samples this first layer of InAs constitutes the seed layer. In the latter case, these InAs are capped with a GaAs spacer layer and a second layer of InAs is deposited.

The GaAs spacer layer is grown at a growth rate $\mathrm{r}_{\mathrm{g}}(\mathrm{Ga})=0.125 \mathrm{ML} / \mathrm{s}$ and $\mathrm{T}_{\mathrm{S}}=510^{\circ} \mathrm{C}$. GaAs spacer layers with thickness of $15 \mathrm{~nm}$ and $20 \mathrm{~nm}$ were fabricated. After 2 minutes of growth interruption for surface flattening, the second InAs layer is deposited. Identical InAs coverage and growth parameters as those used for the growth of the seed layer are employed.

Samples for morphological characterization are cooled down and taken out from the MBE system, while samples for optical studies are capped with a $125 \mathrm{~nm}$ GaAs layer. The first $15 \mathrm{~nm}$ of the capping layer are grown at $\mathrm{r}_{\mathrm{g}}(\mathrm{Ga})=0.125 \mathrm{ML} / \mathrm{s}$ with $\mathrm{F}_{\mathrm{eq}}(\mathrm{As})=0.5 \mathrm{ML} / \mathrm{s}$ at $\mathrm{T}_{\mathrm{S}}=510^{\circ} \mathrm{C}$. Then, substrate temperature is increased to $\mathrm{T}_{\mathrm{S}}=580^{\circ} \mathrm{C}$ and remaining capping layer is completed at $\mathrm{r}_{\mathrm{g}}(\mathrm{Ga})=0.5 \mathrm{ML} / \mathrm{s}$ with $\mathrm{F}_{\mathrm{eq}}(\mathrm{As})=2 \mathrm{ML} / \mathrm{s}$.

Morphological characterization of samples surface was performed by AFM using the same Nanotec set-up employed for substrate patterning. Nanosensors Si AFM tips with nominal tip radius of $7 \mathrm{~nm}$ were employed. $\mu \mathrm{PL}$ characterization of single quantum dots was performed at $4 \mathrm{~K}$ using a diffraction limited confocal microscope inserted in a low vibration cryogen free cryostat (Attocube Attodry 1000). Scanning $\mu$ PL maps were also recorded for identification of the patterned regions using a XY piezo scanner. A $785 \mathrm{~nm}$ continuous wave diode laser was used to excite the photoluminescence which was dispersed by a $750 \mathrm{~mm}$ focal length spectrometer at a resolution of $\sim 30 \mu \mathrm{eV}$ and detected with a cooled Si Charge Coupled Device 
(CCD). The neutral exciton fine structure splitting was measured through its linear polarization angle dependent spectrum.

\section{Results and discussion}

Figure 1 presents nanoholes corresponding to the pattern state right before InAs seed layer SCQDs growth (1a) and before InAs growth for single layer SCQDs (1b). Figure 1a presents a pattern nanohole at the re-growth interface after the thermal ramp up to $\mathrm{T}_{\mathrm{S}}=530^{\circ} \mathrm{C}$ and subsequent cooling down to $\mathrm{T}_{\mathrm{S}}=510^{\circ} \mathrm{C}$. Figure $1 \mathrm{~b}$ presents a pattern nanohole after the growth of a 30nm thick GaAs buffer layer and the thermal ramp up to $\mathrm{T}_{\mathrm{S}}=530^{\circ} \mathrm{C}$ and subsequent cooling down to $\mathrm{T}_{\mathrm{S}}=510^{\circ} \mathrm{C}$. AFM images and profiles along [110] (red line) and [1-10] (blue line) are presented. Original oxide profiles (black line) are included as references.

Nanoholes obtained after HF wet etching replicate geometric oxide dimensions [36]. The nanohole geometry still remains approximately equal to that of the corresponding oxide motif when GaAs buffer layer hasn't been grown (1a). From AFM measurements only a slight evolution of lateral dimensions of the nanohole is observed, with an average increment of $10 \%$ in the width along [110] crystallographic direction and an average reduction of $2 \%$ in the width along [1-10] crystallographic direction. In contrast, when a GaAs buffer layer is grown a strong evolution of the nanohole geometry is observed, leading to shallow motifs, elongated along [110] direction (1b). This is a general behavior found on the overgrowth of nanoholes by MBE, caused by the growth anisotropy $[36,28]$. The lack of buffer layer for vertically stacked InAs SCQDs preserves well the initial pattern nanohole geometry in contrast with the rapid evolution of patterned motifs when GaAs buffer layer is grown [37], spoiling the reproducibility and control of the subsequent InAs nucleation process as it will be shown later.

Figure 2 presents $3,5 \times 3,5 \mu \mathrm{m}^{2}$ AFM images ( $2 \times 2$ pattern motif areas) corresponding to seed layer InAs SCQD grown directly in the re-growth interface, which constitutes the seed nuclei for vertically stacked InAs SCQDs (2a) and single layer InAs SCQD grown on 30nm buffer layer (2c). Identical parameters for InAs growth were employed in both samples $\left(\mathrm{T}_{\mathrm{S}}=510^{\circ} \mathrm{C}\right.$, $\mathrm{F}_{\mathrm{eq}}(\mathrm{As})=0.5 \mathrm{ML} / \mathrm{s}, 1.5 \mathrm{ML}$ InAs). Figure 2a clearly shows that selective InAs QD formation is achieved within the patterned substrate nanoholes; self-assembled QDs don't appear at interstitial positions even without previous growth of GaAs buffer layer, proving the validity of the used procedure for epitaxial re-growth of patterned substrates. Statistics of the number of QD obtained per pattern site is presented in figure $2 b$, showing that very high (98\%) single QD formation per pattern site is achieved as expected from the growth parameters and the size range of the pattern motifs employed [38]. In contrast, high ratio of empty holes or multiple InAs QD formation is obtained on the shallow pattern motifs obtained after the growth of a $30 \mathrm{~nm} \mathrm{GaAs}$ buffer layer, as shown in figure 2c. Statistics of the number of QD obtained per pattern site is 
presented in figure $2 \mathrm{~d}$, showing the loss of both pattern selectivity for InAs nucleation and single QD formation control. The smoothening and elongation of the pattern motifs favors the multiple QD formation within nanoholes [36]. In this sense, Zallo et al. have reported on the growth of QDs pair on nanoholes fabricated by As debt epitaxy when GaAs buffer layers are grown and single QD when no buffer layer is grown [39].

Figure 3 presents 3,5x3,5 $\mu \mathrm{m}^{2}$ AFM images (2x2 pattern motif areas) of vertically stacked InAs SCQD active layer grown on $15 \mathrm{~nm}$ (3a) and 20nm (3c) thick GaAs spacer layer. The obtained statistics of QD number per pattern site are shown in figure $3 \mathrm{~b}$ and 3d. Sample with $15 \mathrm{~nm}$ thick GaAs spacer layer preserves the pattern design with high fidelity and shows $89 \%$ of pattern sites occupied by single InAs SCQD, closely replicating the single QD occupation on the seed layer (figure 2b). However, sample with $20 \mathrm{~nm}$ thick GaAs spacer layer shows $75 \%$ of the pattern sites empty (figure $3 b$ ). This is due to the vanishing of the strain field at the GaAs spacer layer surface. The optimal value obtained for the spacer layer thickness around $15 \mathrm{~nm}$ is largely determined by the size of the SCQDs at the seed layer used in this work. Bigger InAs nuclei (with similar composition) would yield strain field extended to longer distances, that would allow high ratio vertical stacking of positioned nanostructures on thicker spacer layers.

The optical emission was analyzed at $4 \mathrm{~K}$ for all samples. Optical characterization of both capped and uncapped sample of vertically stacked InAs SCQDs with $15 \mathrm{~nm}$ thick GaAs spacer layer has been performed. The uncapped sample was used to study the nanostructures directly grown on the patterned surface (seed layer). The role of this layer is to act as a strain template for nanostructure vertical stacking and poor optical efficiency is expected. Emission from the seed layer is only found under high excitation power $\left(\mathrm{P}_{\text {exc }}\right)$, as presented in figure $4 \mathrm{a}$ for two different pattern sites. The emission is weak and distributed over a very broad energy range where no individual peaks can be identified. In contrast, measuring under the same conditions two pattern sites of the capped sample, we find intense, narrow and well resolved peaks arising from excited shell emission and ground shell charged excitons and biexcitons as shown in figure $4 \mathrm{~b}$. The result suggests that the emission of the seed layer in the capped sample will be negligible at low excitation powers and allow us to evaluate the optical quality of the vertically stacked InAs SCQDs in the active layer in the following.

Figure 5a shows a $\mu \mathrm{PL}$ map showing a region of $6 \times 7$ pattern sites in the vertically stacked InAs SCQDs capped sample. In order to identify all optically active InAs SCQDs, high excitation power was used and optical emission from $900 \mathrm{~nm}$ up to detector cutoff ( 1040 nm) was integrated. The photoluminescence study reveals that $\sim 85 \%$ of the pattern sites are occupied by optically active nanostructures, which is in good agreement with the pattern occupancy evaluated by AFM for samples with $15 \mathrm{~nm}$ thick GaAs spacer layer (figure $3 \mathrm{~b}$ ). The average 
emission energy for the whole array is centered at $1.247 \mathrm{eV}$, with a standard deviation of 23 meV. Partial capping and annealing method could be used to improve the optical emission energy uniformity as it has been demonstrated for self-assembled [40,41] and site-controlled QDs [32,42].

As a figure of merit we have analyzed the spectral line-width of single emission peaks in more than 50 InAs SCQDs under low excitation power $\left(\mathrm{P}_{\mathrm{exc}} \sim 12-40 \mathrm{nW}\right)$ [11]. The full width at half maximum (FWHM) is extracted from gaussian fits and represented in a histogram plot in figure $5 \mathrm{~b}$. The median, average and standard deviation values of the line-width are $146 \mu \mathrm{eV}, 156 \mu \mathrm{eV}$ and $50 \mu \mathrm{eV}$, respectively. Inset shows the spectrum of the InAs SCQD with the lowest FWHM value, $64 \mu \mathrm{V}$, achieved in this work. These values represent a great improvement with respect to our previous work on site-controlled InAs nanostructures [33] where the SCQDs showed an average FWHM $870 \mu \mathrm{eV}$. This fivefold reduction has been obtained without increasing the distance between the optically active InAs SCQDs and the re-growth interface (15 nm in both cases) and highlights the crucial importance of the highest temperature that undergone the regrowth interface. In that previous work this temperature was $\mathrm{T}_{\mathrm{S}}=490^{\circ} \mathrm{C}$ instead of $\mathrm{T}_{\mathrm{S}}=530^{\circ} \mathrm{C}$ employed in this work. Furthermore, similar optical characterization of the nanostructures grown on a $30 \mathrm{~nm}$ thick GaAs buffer layer, as the ones presented in figure $2 \mathrm{c}$, shows average FWHM value of $304 \mu \mathrm{eV}$ and median FWHM value of $273 \mu \mathrm{eV}$, even though the distance from the re-growth interface is twice that of the vertically stacked InAs SCQDs sample. Ignoring this parameter (distance from re-growth interface) the only difference between the samples is the highest temperature the re-growth interface is exposed, being $\mathrm{T}_{\mathrm{S}}=450^{\circ} \mathrm{C}$ for single layer InAs SCQDs instead $\mathrm{T}_{\mathrm{S}}=530{ }^{\circ} \mathrm{C}$ for vertically stacked InAs SCQDs samples. These results clearly show that the thermal treatment of the re-growth interface is more crucial than increasing the distance of the active nanostructures from re-growth interface for improving the SCQDs optical quality.

The longer annealing time at a higher temperature used in this work for vertically stacked InAs samples prior to onset of epitaxial re-growth on the patterned surface strongly reduces spectral diffusion effects caused by the presence of charge carriers at interfaces [26,27,43,44]. In addition, the removal of GaAs buffer layer growth step prior to InAs seed layer deposition preserves the initial geometry of the engraved motifs, which allow us to control the InAs nucleation process obtaining a high single QD formation ratio.

The line-widths found here are of the same order as those reported for InAs SCQDs grown near to re-growth interface on nanohole patterned substrates fabricated by different lithographic techniques $[11,12,45]$. Higher annealing temperature or longer atomic hydrogen treatments of the re-growth interface than used in this work [23] will be crucial to further reduce spectral 
diffusion effects and to improve the optical quality. In this sense, Jöns et al. have demonstrated the growth of InAs SCQDs with a median optical line-width value of $13 \mu \mathrm{eV}$ in e-beam lithography patterned substrates located only at $22 \mathrm{~nm}$ from the re-growth interface that match the optical properties of self-assembled InAs QD [32]. They use a short thermal anneal of the re-growth interface up to $570^{\circ} \mathrm{C}$ and a $10 \mathrm{~nm}$ thick buffer layer. In that work the single QD nucleation per pattern site ratio was around $20 \%$, with a high probability of empty pattern sites or multiple QD formation, evidencing that the growth of high-quality InAs SCQDs with high yield and uniformity is still challenging nowadays. So the procedure presented in this work is a relevant result, simultaneously improving the optical quality of the InAs SCQDs and controlling the single QD occupation ratio. It shows the relevance of the re-growth interface preparation process and proves the highest temperature the re-growth interface is exposed as a crucial parameter in the surface preparation step of patterned substrates. Moreover, the developed process has a great room for improvement by increasing the temperature of thermal treatment of re-growth interface above $530^{\circ} \mathrm{C}$ and/or by introducing $\mathrm{AlGaAs}$ blocking layers between regrowth interface and the SCQD active layer.

Figure 6 shows a more detailed optical characterization performed in two InAs SCQDs from the vertically stacked sample, labeled in the following QD1 and QD2. The excitation power $\left(\mathrm{P}_{\mathrm{exc}}\right)$ dependence of the $\mu \mathrm{PL}$ integrated intensity (not shown) of the main peaks has been fitted to a power law $\mathrm{I}_{\mu \mathrm{PL}}=\alpha \mathrm{P}_{\mathrm{exc}}{ }^{\mathrm{m}}$ as usual $[46,47]$. At low powers, QD1 emission is dominated by a single peak at $1.2798 \mathrm{eV}$, with FWHM $115 \mu \mathrm{eV}$ and exponent $\mathrm{m}=1.12 \pm 0.03$, which is attributed to the neutral exciton $\mathrm{X}$. With increasing excitation power the spectrum becomes dominated by a peak at $1.2782 \mathrm{eV}$ with exponent $\mathrm{m}=1.80 \pm 0.08$ attributed to the neutral biexciton $\mathrm{XX}$. Angle resolved linear polarization measurements (figure 6c) reveals that both $\mathrm{X}$ and $\mathrm{XX}$ are indeed two linearly polarized doublets split by the electron-hole anisotropic exchange interaction as expected for InAs QDs on GaAs (001) [48]. The fine structure splitting (FSS) is clearly resolved with a value $82 \pm 2 \mu \mathrm{eV}$. The two other peaks shifted in energy from the neutral exciton by 3.1 $\mathrm{meV}(1.2829 \mathrm{eV})$ and $-6.8 \mathrm{meV}(1.2730 \mathrm{eV})$ show no polarization splitting and present exponents $m=1.20 \pm 0.03$ and $m=1.41 \pm 0.02$, respectively. They are attributed to positively and negatively charged trions $\mathrm{X}^{+}$and $\mathrm{X}^{-}$[49] with slopes different than one due to the carrier trapping dynamics [47].

The same analysis for QD2 emission shows that the peaks at $1.2361 \mathrm{eV}$ and $1.2386 \mathrm{eV}$ have exponents $\mathrm{m}=2.01 \pm 0.15$ and $\mathrm{m}=1.16 \pm 0.03$ and polarization resolved measurements consistent with neutral biexciton XX and exciton X recombination, respectively. A FSS value of $42 \pm 3 \mu \mathrm{eV}$ was found in this case. The emission spectrum however is dominated from the low excitation regime up to saturation by a peak at $1.2341 \mathrm{eV}$ with FWHM $95 \mu \mathrm{eV}$ which shows no 
polarization dependence and, therefore, is attributed to a negative trion $\mathrm{X}^{-}$arising from a more efficient charging process in this QD.

The FSS values found for QD1 and QD2 are comparable to those found in self assembled InAs QDs emitting at similar energies [50]. It shall be noticed that the energy splittings have opposite sign in both QDs with the low energy peak of the doublet linearly polarized along different directions in each QD (almost 90 degrees shift). QD confinement anisotropy (elongation), strain, and piezoelectric effects as well as intrinsic atomistic asymmetries or extrinsic (caused by In/Ga alloying for instance) have to be analyzed in detail to explain this result but are beyond of the scope of this work [50,51]. A reduction of the FSS value with increasing exciton emission energy and decreasing QD size has been observed for self-assembled QDs [50,52]. In this sense, the use of a partial capping and annealing method or post-growth annealing treatments to limit the QD size could be useful to obtain smaller InAs SCQD with expected reduced FSS values that could be used to develop deterministic entangled photon pair sources.

The capability, shown in this work, of simultaneously improving the optical quality of InAs SCQDs and retaining high single occupation ratio, proves the highest temperature the re-growth interface is exposed as a crucial parameter for re-growth interface cleanliness, making more attainable the technological goal of developing a high yield growth process for highly uniform site controlled nanostructures with improved optical properties.

\section{Conclusion}

In summary, the growth and optical characterization of single InAs SCQDs grown on GaAs (001) substrates patterned by AFM oxidation lithography has been reported. InAs SCQDs directly grown on the patterned surface were used as a seed layer and strain template for the nucleation of optically active single InAs SCQDs with high probability using optimized growth parameters, resulting in a fivefold reduction of the exciton line-width from $870 \mu \mathrm{eV}$ to $156 \mu \mathrm{eV}$ for these QDs. The preservation of initial geometry of the engraved pattern motifs after the regrowth interface preparation process, the lack of buffer layer growth prior to InAs seed layer deposition and the development of suitable growth conditions provide us an improvement of SCQDs active layer optical properties retaining at the same time a high ratio of single occupation $(89 \%)$. The presented results demonstrate the highest temperature the re-growth interface is exposed as a crucial parameter in the surface preparation step of patterned substrates. Higher temperature and longer treatments of the re-growth interface than the employed in this work would make more attainable the development of a high yield growth process for uniform site controlled nanostructures with improved optical properties. 


\section{Acknowledgment}

The authors thank financial support by Spanish MINECO through grants ENE2012-37804-C0202 and TEC2011-29120-C05-04. Jesús Herranz acknowledges to the JAE program for the funds.

\section{References}

[1] Santori C, Pelton M, Solomon G, Dale Y and Yamamoto Y 2001 Triggered Single Photons from a Quantum Dot Phys. Rev. Lett. 86 1502-5

[2] Yuan Z, Kardynal B E, Stevenson R M, Shields A J, Lobo C J, Cooper K, Beattie N S, Ritchie D A and Pepper M 2002 Electrically Driven Single-Photon Source Science 295 $102-5$

[3] Stevenson R M, Young R J, Atkinson P, Cooper K, Ritchie D A and Shields A J 2006 A semiconductor source of triggered entangled photon pairs Nature 439 179-82

[4] Salter C L, Stevenson R M, Farrer I, Nicoll C A, Ritchie D A and Shields A J 2010 An entangled-light-emitting diode Nature 465 594-7

[5] Kiravittaya S, Rastelli A and Schmidt O G 2009 Advanced quantum dot configurations Rep. Prog. Phys. 72046502

[6] Dalacu D, Reimer M e., Fréderick S, Kim D, Lapointe J, Poole P j., Aers G c., Williams R 1., Ross McKinnon W, Korkusinski M and Hawrylak P 2010 Directed self-assembly of single quantum dots for telecommunication wavelength optical devices Laser Photonics Rev. 4 283-99

[7] Lan H and Ding Y 2012 Ordering, positioning and uniformity of quantum dot arrays Nano Today 7 94-123

[8] Yang B, Liu F and Lagally M G 2004 Local Strain-Mediated Chemical Potential Control of Quantum Dot Self-Organization in Heteroepitaxy Phys. Rev. Lett. 92025502

[9] Kiravittaya S, Rastelli A and Schmidt O G 2005 Self-assembled InAs quantum dots on patterned GaAs(001) substrates: Formation and shape evolution Appl. Phys. Lett. 87 243112

[10] Mereni L O, Dimastrodonato V, Young R J and Pelucchi E 2009 A site-controlled quantum dot system offering both high uniformity and spectral purity Appl. Phys. Lett. 94 223121

[11] Schneider C, Huggenberger A, Gschrey M, Gold P, Rodt S, Forchel A, Reitzenstein S, Höfling S and Kamp M 2012 In(Ga)As/GaAs site-controlled quantum dots with tailored morphology and high optical quality Phys. Status Solidi A 209 2379-86

[12] Skiba-Szymanska J, Jamil A, Farrer I, Ward M B, Nicoll C A, Ellis D J P, Griffiths J P, Anderson D, Jones G A C, Ritchie D A and Shields A J 2011 Narrow emission linewidths of positioned InAs quantum dots grown on pre-patterned GaAs(100) substrates Nanotechnology 22065302

[13] Helfrich M, Hu D Z, Hendrickson J, Gehl M, Rülke D, Gröger R, Litvinov D, Linden S, Wegener M, Gerthsen D, Schimmel T, Hetterich M, Kalt H, Khitrova G, Gibbs H M and 
Schaadt D M 2011 Growth and annealing of InAs quantum dots on pre-structured GaAs substrates J. Cryst. Growth 323 187-90

[14] Schramm A, Tommila J, Strelow C, Hakkarainen T V, Tukiainen A, Dumitrescu M, Mews A, Kipp T and Guina M 2012 Large array of single, site-controlled InAs quantum dots fabricated by UV-nanoimprint lithography and molecular beam epitaxy Nanotechnology 23175701

[15] Cheng C-C, Meneou K and Cheng K Y 2011 Effects of nano-pattern size on the property of InAs site-controlled quantum dots J. Cryst. Growth 323 180-2

[16] Song H Z, Usuki T, Ohshima T, Sakuma Y, Kawabe M, Okada Y, Takemoto K, Miyazawa T, Hirose S, Nakata Y, Takatsu M and Yokoyama N 2006 Site-controlled quantum dots fabricated using an atomic-force microscope assisted technique Nanoscale Res. Lett. 1 160-6

[17] Martín-Sánchez J, Muñoz-Matutano G, Herranz J, Canet-Ferrer J, Alén B, González Y, Alonso-González P, Fuster D, González L, Martínez-Pastor J and Briones F 2009 Single Photon Emission from Site-Controlled InAs Quantum Dots Grown on GaAs(001) Patterned Substrates ACS Nano 3 1513-7

[18] Cha K M, Horiuchi I, Shibata K and Hirakawa K 2012 Size-Limiting Effect of SiteControlled InAs Quantum Dots Grown at High Temperatures by Molecular Beam Epitaxy Appl. Phys. Express 5085501

[19] Prieto I, Herranz J, Gonzalez Y, Canet-Ferrer J, Wewior L, Postigo P ., Alen B, Gonzalez L, Kaldirim M, Munioz-Camuniez L, Fuster D, Taboada A G, Ripalda J M, Briones F, Munoz-Matutano G and Martinez-Pastor J 2011 Different strategies towards the deterministic coupling of a single Quantum Dot to a photonic crystal cavity mode 2011 13th International Conference on Transparent Optical Networks (ICTON) 2011 13th International Conference on Transparent Optical Networks (ICTON) pp 1-5

[20] Prieto I, Herranz J, Wewior L, González Y, Alén B, González L and Postigo P A 2013 High quality factor GaAs-based photonic crystal microcavities by epitaxial re-growth $O p t$. Express 21 31615-22

[21] Bastiman F and Cullis A G $2010 \mathrm{GaAs}(000$ 1) planarization after conventional oxide removal utilising self-governed InAs QD site selection Appl. Surf. Sci. 256 4269-71

[22] Bell G R, Kaijaks N S, Dixon R J and McConville C F 1998 Atomic hydrogen cleaning of polar III-V semiconductor surfaces Surf. Sci. 401 125-37

[23] Martín-Sánchez J, González Y, Alonso-González P and González L 2008 Improvement of InAs quantum dots optical properties in close proximity to $\operatorname{GaAs}\left(\begin{array}{lll}0 & 0 & 1\end{array}\right)$ substrate surface J. Cryst. Growth 310 4676-80

[24] Atkinson P and Schmidt O G 2009 Gallium-assisted deoxidation of patterned substrates for site-controlled growth of InAs quantum dots J. Cryst. Growth 311 1815-8

[25] Tommila J, Tukiainen A, Viheriälä J, Schramm A, Hakkarainen T, Aho A, Stenberg P, Dumitrescu M and Guina M 2011 Nanoimprint lithography patterned GaAs templates for site-controlled InAs quantum dots J. Cryst. Growth $\mathbf{3 2 3} 183-6$

[26] Bayer M and Forchel A 2002 Temperature dependence of the exciton homogeneous linewidth in In0.60Ga0.40As/GaAs self-assembled quantum dots Phys. Rev. B 65041308 
[27] Wang C F, Badolato A, Wilson-Rae I, Petroff P M, Hu E, Urayama J and Imamoğlu A 2004 Optical properties of single InAs quantum dots in close proximity to surfaces Appl. Phys. Lett. 85 3423-5

[28] Yakes M K, Yang L, Bracker A S, Sweeney T M, Brereton P G, Kim M, Kim C S, Vora P M, Park D, Carter S G and Gammon D 2013 Leveraging Crystal Anisotropy for Deterministic Growth of InAs Quantum Dots with Narrow Optical Linewidths Nano Lett. $134870-5$

[29] Nakamura Y, Schmidt O G, Jin-Phillipp N Y, Kiravittaya S, Müller C, Eberl K, Gräbeldinger H and Schweizer H 2002 Vertical alignment of laterally ordered InAs and InGaAs quantum dot arrays on patterned ( $\left.\begin{array}{lll}0 & 0 & 1\end{array}\right)$ GaAs substrates J. Cryst. Growth 242 339-44

[30] Huggenberger A, Heckelmann S, Schneider C, Höfling S, Reitzenstein S, Worschech L, Kamp M and Forchel A 2011 Narrow spectral linewidth from single site-controlled In(Ga)As quantum dots with high uniformity Appl. Phys. Lett. 98131104

[31] Mohan A, Felici M, Gallo P, Dwir B, Rudra A, Faist J and Kapon E 2010 Polarizationentangled photons produced with high-symmetry site-controlled quantum dots Nat. Photonics 4 302-6

[32] Jöns K D, Atkinson P, Müller M, Heldmaier M, Ulrich S M, Schmidt O G and Michler P 2013 Triggered Indistinguishable Single Photons with Narrow Line Widths from SiteControlled Quantum Dots Nano Lett. 13 126-30

[33] Canet-Ferrer J, Munoz-Matutano G, Herranz J, Rivas D, Alen B, Gonzalez Y, Fuster D, Gonzalez L and Martínez-Pastor J 2013 Exciton and multiexciton optical properties of single InAs/GaAs site-controlled quantum dots Appl. Phys. Lett. 103183112

[34] Briones F, González L and Ruiz A 1989 Atomic layer molecular beam epitaxy (Almbe) of III-V compounds: Growth modes and applications Appl. Phys. A 49 729-37

[35] Garcia J C, Neri C and Massies J 1989 A comparative study of the interaction kinetics of As2 and As4 molecules with Ga-rich GaAs (001) surfaces J. Cryst. Growth 98 511-8

[36] Martín-Sánchez J, Alonso-González P, Herranz J, González Y and González L 2009 Sitecontrolled lateral arrangements of InAs quantum dots grown on GaAs(001) patterned substrates by atomic force microscopy local oxidation nanolithography Nanotechnology 20125302

[37] Heidemeyer H, Müller C and Schmidt O G 2004 Highly ordered arrays of In(Ga)As quantum dots on patterned GaAs ( $\left.\begin{array}{lll}0 & 0 & 1\end{array}\right)$ substrates J. Cryst. Growth $261444-9$

[38] Herranz J, González L, Wewior L, Alén B, Fuster D and González Y 2014 Study of Growth Parameters for Single InAs QD Formation on GaAs(001) Patterned Substrates by Local Oxidation Lithography Cryst. Growth Des. 15 666-72

[39] Zallo E, Atkinson P, Rastelli A and Schmidt O G 2012 Controlling the formation of quantum dot pairs using nanohole templates J. Cryst. Growth 338 232-8

[40] Garcia J M, Mankad T, Holtz P O, Wellman P J and Petroff P M 1998 Electronic states tuning of InAs self-assembled quantum dots Appl. Phys. Lett. 72 3172-4 
[41] Wang L, Rastelli A and Schmidt O G 2006 Structural and optical properties of $\mathrm{In}(\mathrm{Ga}) \mathrm{As} / \mathrm{GaAs}$ quantum dots treated by partial capping and annealing J. Appl. Phys. 100 064313

[42] Huggenberger A, Schneider C, Drescher C, Heckelmann S, Heindel T, Reitzenstein S, Kamp M, Höfling S, Worschech L and Forchel A 2011 Site-controlled In(Ga)As/GaAs quantum dots for integration into optically and electrically operated devices J. Cryst. Growth 323 194-7

[43] Abbarchi M, Troiani F, Mastrandrea C, Goldoni G, Kuroda T, Mano T, Sakoda K, Koguchi N, Sanguinetti S, Vinattieri A and Gurioli M 2008 Spectral diffusion and line broadening in single self-assembled GaAs/AlGaAs quantum dot photoluminescence Appl. Phys. Lett. 93162101

[44] Berthelot A, Favero I, Cassabois G, Voisin C, Delalande C, Roussignol P, Ferreira R and Gérard J M 2006 Unconventional motional narrowing in the optical spectrum of a semiconductor quantum dot Nat. Phys. 2 759-64

[45] Tommila J, Schramm A, Hakkarainen T V, Dumitrescu M and Guina M 2013 Sizedependent properties of single InAs quantum dots grown in nanoimprint lithography patterned GaAs pits Nanotechnology 24235204

[46] Grundmann M and Bimberg D 1997 Theory of random population for quantum dots Phys. Rev. B 55 9740-5

[47] Gomis-Bresco J, Muñoz-Matutano G, Martínez-Pastor J, Alén B, Seravalli L, Frigeri P, Trevisi G and Franchi S 2011 Random population model to explain the recombination dynamics in single InAs/GaAs quantum dots under selective optical pumping New J. Phys. 13023022

[48] Stevenson R M, Thompson R M, Shields A J, Farrer I, Kardynal B E, Ritchie D A and Pepper M 2002 Quantum dots as a photon source for passive quantum key encoding Phys. Rev. B 66081302

[49] Rodt S, Schliwa A, Pötschke K, Guffarth F and Bimberg D 2005 Correlation of structural and few-particle properties of self-organized InAs/GaAs quantum dots Phys. Rev. B 71 155325

[50] Seguin R, Schliwa A, Rodt S, Pötschke K, Pohl U W and Bimberg D 2005 SizeDependent Fine-Structure Splitting in Self-Organized InAs/GaAs Quantum Dots Phys. Rev. Lett. 95257402

[51] Singh R and Bester G 2010 Lower Bound for the Excitonic Fine Structure Splitting in Self-Assembled Quantum Dots Phys. Rev. Lett. 104196803

[52] Young R J, Stevenson R M, Shields A J, Atkinson P, Cooper K, Ritchie D A, Groom K M, Tartakovskii A I and Skolnick M S 2005 Inversion of exciton level splitting in quantum dots Phys. Rev. B 72113305 


\section{Figures}

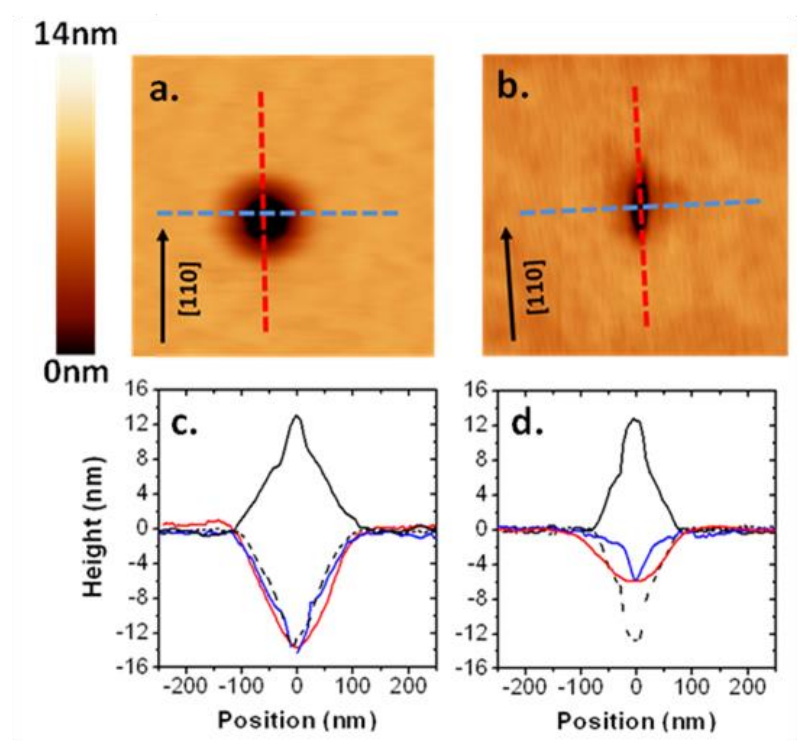

Figure $1-600 x 600 \mathrm{~nm}^{2}$ AFM image of pattern nanoholes obtained after and thermal ramp up to $\mathrm{T}_{\mathrm{S}}=530^{\circ} \mathrm{C}$ and subsequent cooling down to $\mathrm{T}_{\mathrm{S}}=510^{\circ} \mathrm{C}$ (a) and after atomic $\mathrm{H}$ treatment, growth of a 30nm thick GaAs buffer layer, thermal ramp up to $\mathrm{T}_{\mathrm{S}}=530^{\circ} \mathrm{C}$ and subsequent cooling down to $\mathrm{T}_{\mathrm{S}}=510^{\circ} \mathrm{C}$ (b). Corresponding $\mathrm{AFM}$ profiles along [110] (red) and [1-10] (blue) crystallographic directions. Original oxide profile (black solid) and negative oxide profile (black dashed) are shown as references. 

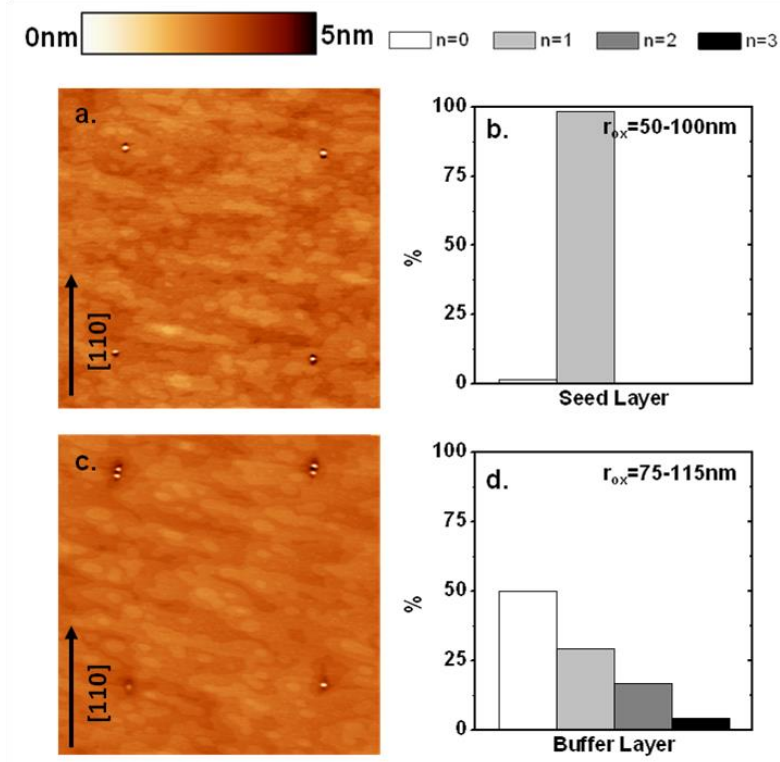

Figure2- $3.5 \times 3.5 \mu \mathrm{m}^{2}$ AFM images, showing $2 \times 2$ pattern sites, of seed layer InAs SCQDs grown without GaAs buffer layer on the patterned substrate surface (a) and InAs SCQDs grown on 30nm thick GaAs buffer layer (c). Occupation histogram plots are presented (b and d). 


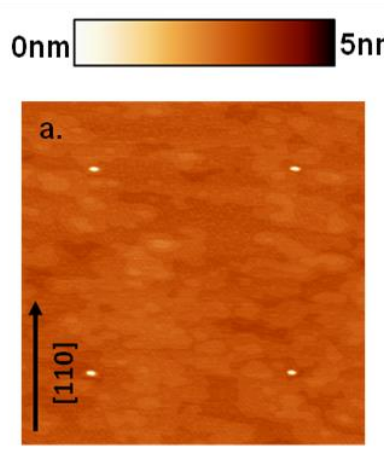

$\mathrm{nm} \square \mathrm{n}=0 \quad \square \mathrm{n}=1 \quad \square \mathrm{n}=2$
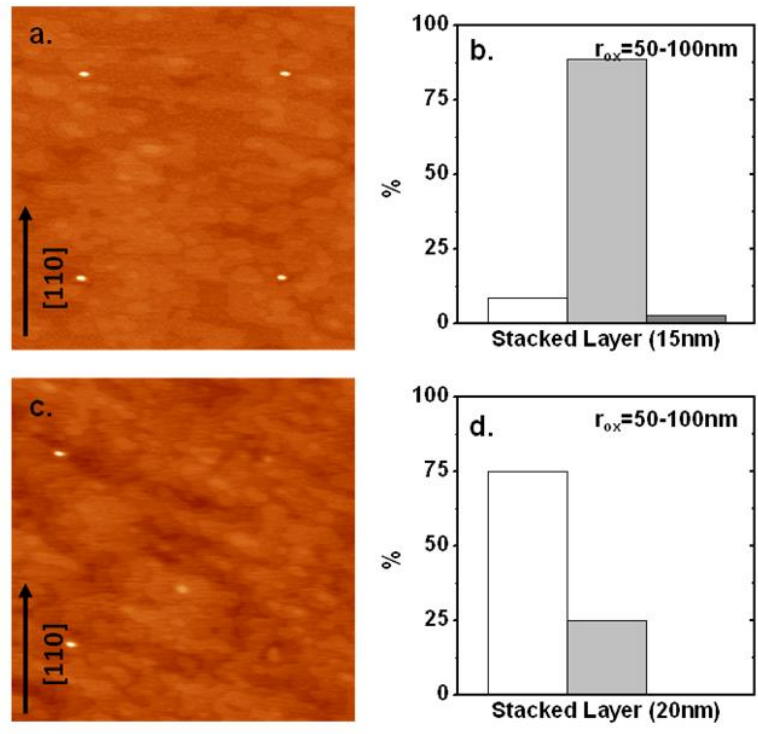

Figure3- $3.5 \times 3.5 \mu \mathrm{m}^{2}$ AFM images, showing $2 \times 2$ pattern sites, of vertically stacked active layer InAs SCQDs with $15 \mathrm{~nm}$ thick GaAs spacer layer (a) and 20nm thick GaAs spacer layer (c). Occupation histogram plots are shown (b and d). 


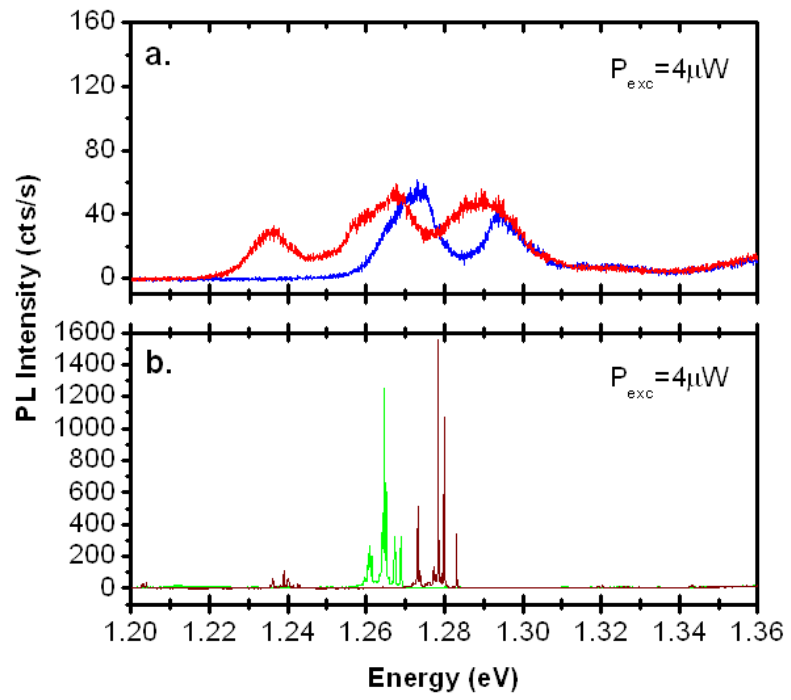

Figure 4- Spectra taken at two pattern sites for InAs QD seed layer (a) and InAs QD active layer (b) at low temperature $\mathrm{T}=4 \mathrm{~K}$. Note the factor 10 in PL intensity scales. 
a.

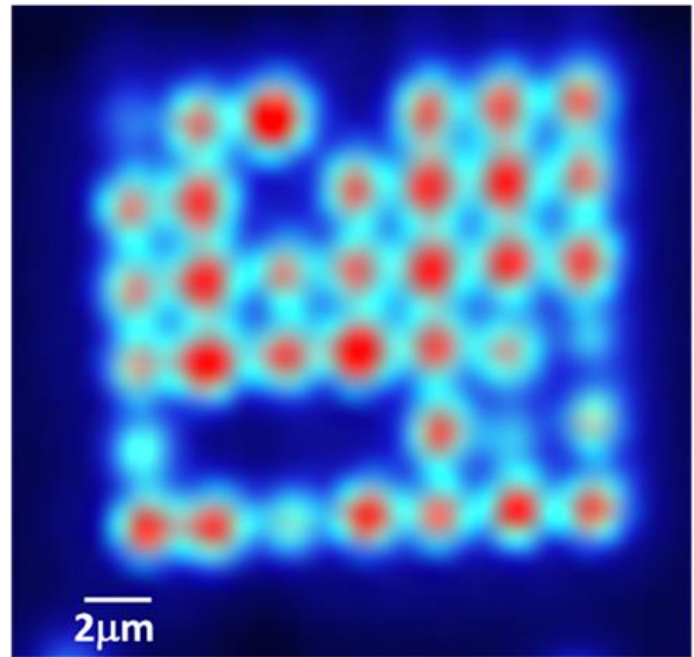

b.

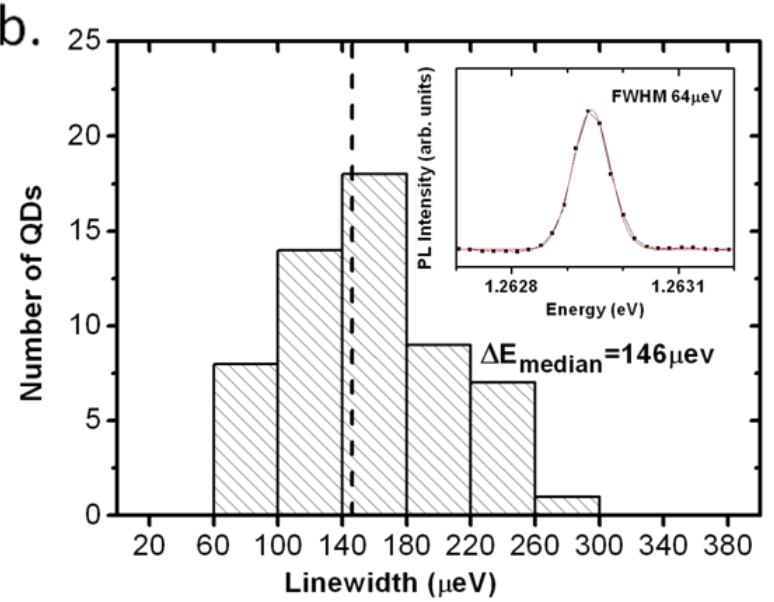

Figure 5 - High excitation power $\mu \mathrm{PL}$ map of patterned region showing $6 \times 7$ pattern sites (a) and optical FWHM line width histogram of 57 InAs SCQDs (b) Inset: single InAs SCQD

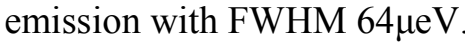


a.

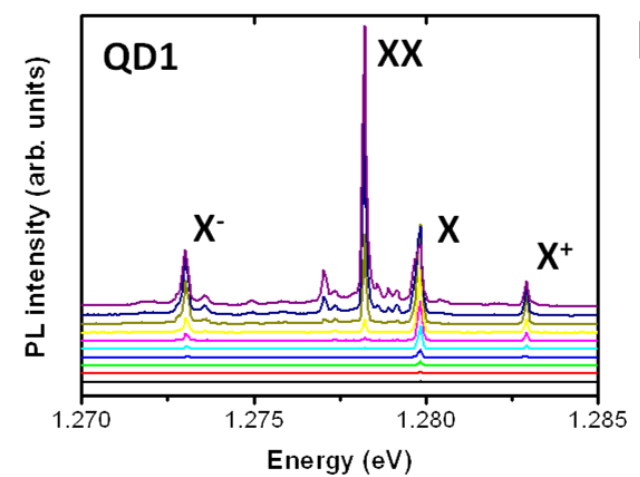

c.

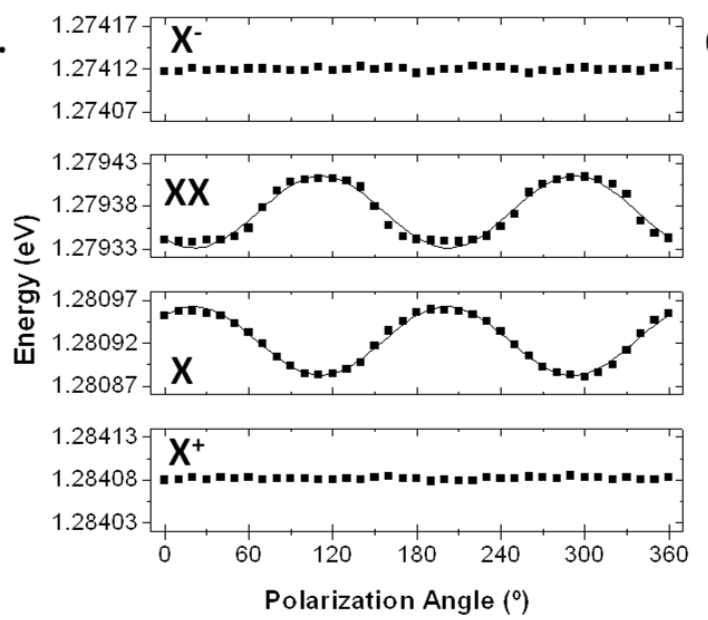

b.

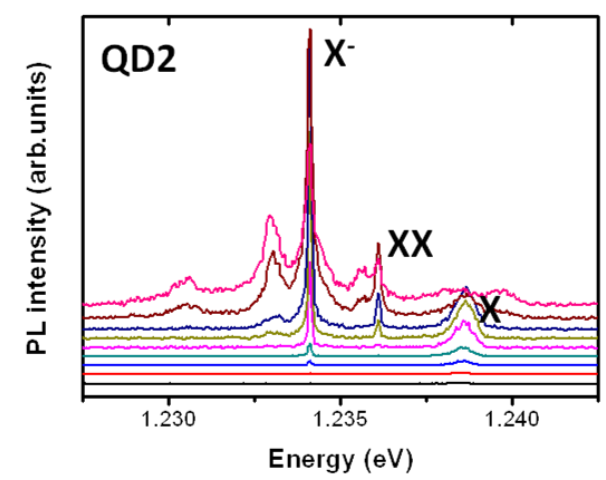

d.
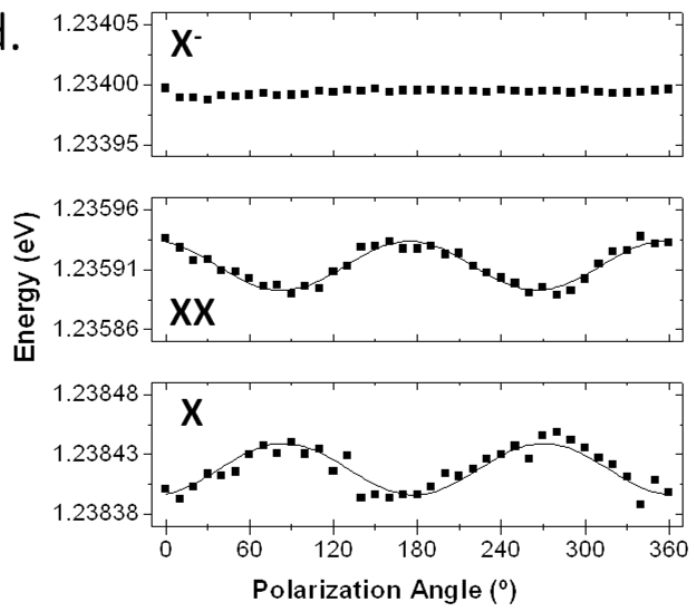

Figure 6 - Optical characterization of two InAs SCQDs. $\mu$ PL spectra for increasing excitation power for QD1 (a) and QD2 (b). Polarization dependent energy position peak for labeled peaks in QD1 (c) and QD2 (d). 